\title{
Expressed sequences tags of the anther smut fungus, Microbotryum violaceum, identify mating and pathogenicity genes Roxana Yockteng1,2, Sylvain Marthey ${ }^{3}$, Hélène Chiapello ${ }^{3}$, Annie Gendrault ${ }^{3}$, Michael E Hood ${ }^{4}$, François Rodolphe ${ }^{3}$, Benjamin Devier ${ }^{1}$, Patrick Wincker ${ }^{5}$, Carole Dossat ${ }^{5}$ and Tatiana Giraud*1
}

Address: ${ }^{1}$ UMR 8079 CNRS-UPS, Ecologie, Systématique et Evolution, Bâtiment 360, Université Paris-Sud, F-91405 Orsay Cedex, France, ${ }^{2}$ UMR 5202, CNRS-MNHN, Origine, Structure et Evolution de la Biodiversité, Département Systématique et Evolution, 16 rue Buffon CP 39, 75005, Paris, France, ${ }^{3}$ INRA, Unité Mathématique, Informatique et Génome, Domaine Vilvert, Jouy-en-Josas, F-78352, France, ${ }^{4}$ Department of Biology, Amherst College, Amherst, MA 01002, USA and ${ }^{5}$ Génoscope, UMR CNRS 8030, 2 Gaston Crémieux, CP 5706, 91507 Evry, France

Email: Roxana Yockteng - yockteng@mnhn.fr; Sylvain Marthey - smarthey@jouy.inra.fr; Hélène Chiapello - helene.chiapello@jouy.inra.fr; Annie Gendrault - annie.jacquemard@jouy.inra.fr; Michael E Hood - Michael.Hood@virginia.edu; François Rodolphe - Francois.Rodolphe@jouy.inra.fr; Benjamin Devier - Benjamin.Devier@u-psud.fr;

Patrick Wincker - pwincker@genoscope.cns.fr; Carole Dossat - cdossat@genoscope.cns.fr; Tatiana Giraud* - Tatiana.Giraud@u-psud.fr

* Corresponding author

Published: 10 August 2007

BMC Genomics 2007, 8:272 doi:10.1 186/1471-2164-8-272
Received: 17 November 2006

Accepted: 10 August 2007

This article is available from: http://www.biomedcentral.com/I47I-2/64/8/272

(c) 2007 Yockteng et al; licensee BioMed Central Ltd.

This is an Open Access article distributed under the terms of the Creative Commons Attribution License (http://creativecommons.org/licenses/by/2.0), which permits unrestricted use, distribution, and reproduction in any medium, provided the original work is properly cited.

\begin{abstract}
Background: The basidiomycete fungus Microbotryum violaceum is responsible for the anther-smut disease in many plants of the Caryophyllaceae family and is a model in genetics and evolutionary biology. Infection is initiated by dikaryotic hyphae produced after the conjugation of two haploid sporidia of opposite mating type. This study describes M. violaceum ESTs corresponding to nuclear genes expressed during conjugation and early hyphal production.

Results: A normalized cDNA library generated 24,128 sequences, which were assembled into 7,765 unique genes; $25.2 \%$ of them displayed significant similarity to annotated proteins from other organisms, $74.3 \%$ a weak similarity to the same set of known proteins, and $0.5 \%$ were orphans. We identified putative pheromone receptors and genes that in other fungi are involved in the mating process. We also identified many sequences similar to genes known to be involved in pathogenicity in other fungi. The $M$. violaceum EST database, MICROBASE, is available on the Web and provides access to the sequences, assembled contigs, annotations and programs to compare similarities against MICROBASE.
\end{abstract}

Conclusion: This study provides a basis for cloning the mating type locus, for further investigation of pathogenicity genes in the anther smut fungi, and for comparative genomics.

\section{Background}

Deciphering the molecular mechanisms involved in infection is important for the control of devastating crop diseases. Furthermore, the comparison of pathogenicityrelated genes from different fungi provides insight into the evolution of host-pathogen interactions, thereby advancing our understanding of host specificity, virulence, and the emergence of new diseases. Modern sequencing technologies have led to a remarkable increase in genomic data available for identifying genes by similar- 
ity searches [1]. Key genes involved in pathogenicity in several fungi have been compiled into the PHI database [2].

In the smut fungi of monocot hosts (e.g. Ustilago maydis and $U$. hordei, major pathogens of corn and barley, respectively), the sexual phase and the genes linked to the mating-type loci play a key role in development and pathogenicity [3]. Mating-type loci determine sexual compatibility: only individuals differing at these loci can mate. In $U$. maydis, cell recognition and fusion is regulated by a pheromone/receptor system that resides at the $a$ locus. After fusion, the dikaryon is maintained and cells switch to filamentous growth if they are heterozygous for the second mating type locus, the $b$ locus $[4,5]$. The $b$ locus encodes two homeodomain proteins that function as transcriptional regulators after dimerization. The majority of sexual basidiomycete fungi possess such a system called "tetrapolar", where $a$ and $b$ unlinked loci (respectively called B and A in some species) are both involved in sexual compatibility and are often multiallelic $[5,6]$. Other members of this phylum are "bipolar", due to the $a$ and $b$ loci being tightly linked (e.g. in $U$. hordei, [7]) or due to one of the two mating type loci having lost their role in mating type specificity (e.g. in Coprinellus disseminatus, [8]). Tetrapolarity is likely ancestral [9] and promotes outcrossing as it increases the number of available mating type. The study of mating-type loci is important for understanding the infection process and the evolution of mating systems in basidiomycetes.

A widely recognized model to study host-pathogen coevolution and fungal genetics is the anther smut fungus Microbotryum violaceum (Pers.) Deml and Oberw. (formerly Ustilago violacea (Pers.) Fuckel), which is a basidiomycete, obligate parasite of more than 100 perennial species of Caryophyllaceae [10]. In plants infected by $M$. violaceum, fungal teliospores are produced in anthers and diseased plants are usually completely sterilized, the pollen being replaced by fungal spores and the stigmas and ovaries being reduced. New infections occur when fungal spores are transported from a diseased to a healthy plant by the insects that usually serve as pollinators. Once deposited on a host plant, diploid teliospores undergo meiosis and give rise to four haploid cells, two of mating type A1 and two of mating-type A2, M. violaceum having a bipolar mating system. Each of these post-meiotic cells can buds off yeast-like sporidia on the plant surface. New infectious dikaryons are produced only after conjugation of two cells of opposite mating-types [11]. The fungus then grows endophytically and causes perennial systemic infections.

Although M. violaceum is related to major crop pathogens like U. maydis and Puccinia spp., it has no impact on human activities, making it valuable for the study of natural host-pathogen coevolution, and avoiding the risk of dispersion in human crops. However, one present limitation of this model is that little genomic sequence data are available, except studies on transposable elements and on the genomic defense mechanism against the accumulation of mobile elements [12]. In particular, the matingtype locus was reticent to several cloning attempts ( $T$. Giraud and M.E. Hood, unpublished; J. Kronstad, pers. com.) and there exist few sequences of expressed genes from M. violaceum in public databases. Only a few Microbotryum genes that contribute to hyphal development and subsequent infectious capability have been described [13].

The generation of Expressed Sequence Tags (EST) is an efficient tool to discover novel genes and investigate their expression at different developmental stages (e.g., $[14,15])$. Therefore, a cDNA library has been built from pools of mating haploid cells and growing infectious hyphae for a single dikaryotic isolate of $\mathrm{M}$. violaceum collected from the host plant species Silene latifolia. Genes involved in mating and during early pathogenic development were expected to be expressed under these conditions because they represent the mating and infectious stages. We generated 24,128 ESTs from this library, on which we performed similarity searches in order to identify genes with functions known as important for these developmental stages.

\section{Results and discussion EST sequence analysis}

The cDNA library created from poly(A)+mRNA from seven days-old mixed A1 and A2 cultures produced enough material to sequence 40,000 clones. A total of 28,430 sequences were obtained (success rate of $71 \%$ ) with an average read length of $815 \mathrm{bp}$, which is similar to the EST library of $U$. maydis [14]. Some ribosomal $(\mathrm{n}=$ 109), mitochondrial $(\mathrm{n}=16)$ and vector $(\mathrm{n}=14)$ sequences were identified. After discarding them, a total of 24,128 ESTs were obtained $(85 \%$ of the initial sequences). After trimming vector and low quality sequences, the average CDNA read was not very long, with $345 \pm 167$ bases (mean \pm SD). We indeed did not select the sizes of mRNA, as recommended for normalized libraries.

These 24,128 ESTs were assembled into 4,178 contigs while 3,587 remained as singlets (Figure 1). This corresponds to a redundancy of $85 \%$ (number of ESTs assembled in clusters/total number of ESTs), which is very high compared to the redundancy obtained in other fungal EST libraries such as Phytophtora parasitica (49.5\%, [16]), Botrytis cinerea $(67 \%,[15])$ or Ustilago maydis $(72.3 \%,[14])$. This does not result from a low efficiency of our normali- 
zation, but from the large scale of the present study compared to the ones cited above. Our library indeed had a size of $6.65 \times$ compared to $P$. parasitica, $3.74 \times$ compared to B. cinerea and $8.4 \times$ compared to $U$. maydis. The number of unisequences (i.e. all contigs and singlets) identified in our library represents 7,765 putative unique genes, which lies within the total gene number in fungi (range from 5,570 to 16,597 [17]).

\section{Online database: MICROBASE}

A website is available with open access to the EST sequences, unisequences and annotations [18]. Several tools are made available, allowing visualising contig assemblies and performing searches on our ESTs or unisequences, by BLAST, by annotation, by function, and by sequence ID. The database was named MICROBASE, after Microbotryum EST database.

\section{Functional classification}

Similarity searches performed on the 7,765 unisequences indicated that $1,953(25.2 \%)$ had a highly significant similarity to UniProt or Genbank entries (E-value $\leq 10^{-10}$ ). Among these, 125 unisequences were similar to strictly "hypothetical" or "unknown" proteins. A total of 818 sequences $(10.5 \%)$ could be classified in a putative cellular function according to the characterization scheme outlined by the Gene Ontology Consortium. In addition, $5,772(74.3 \%)$ unisequences showed moderate to very low similarity $\left(10^{-10}<\right.$ E-value $\left.<10^{-1}\right)$ to the UniProt and
Genbank databases. A total of 125 unisequences (0.5\%) had no similarity to any existing UniProt nor Genbank entry ("orphans"). This high frequency of genes without significant BLAST hit is similar to previous fungal EST libraries (e.g., $[14,15])$. In some cases, this lack of similarity to protein database entries could be due to the sequence being derived from the $5^{\prime}$ or $3^{\prime}$ untranslated region of the cDNA [19]. Among the 1,953 sequences that had a highly significant similarity to Genbank entries, 93.48\% had their most significant hit against fungal sequences and $4.79 \%$ against sequences from other organisms: animals $(1.6 \%)$, plants $(2.58 \%)$, protozoa $(0.37 \%)$ and bacteria $(0.24 \%)$.

Regarding the Gene Ontology classes in our M. violaceum EST library, the molecular function class was the most abundant $(37.65 \%)$, followed by the cellular component class $(30.68 \%)$, and by the biological process class $(16.35 \%)$. Whitin the molecular function class, sequences classified in catalytic and binding activities functions were the most abundant (Figure 2). We found also 33 unisequences with significant similarity to genes belonging to, or linked to, the mating-type loci of other basidiomycetes (see the Table in Manual Annotations in the "Annotations" section at MICROBASE) and 70 unisequences with significant similarity to genes that have been shown experimentally to be involved in pathogenicity in other fungi according to the PHI-base [2] (Table 1, see also Manual Annotations in the "Annotations" section at MICRO-

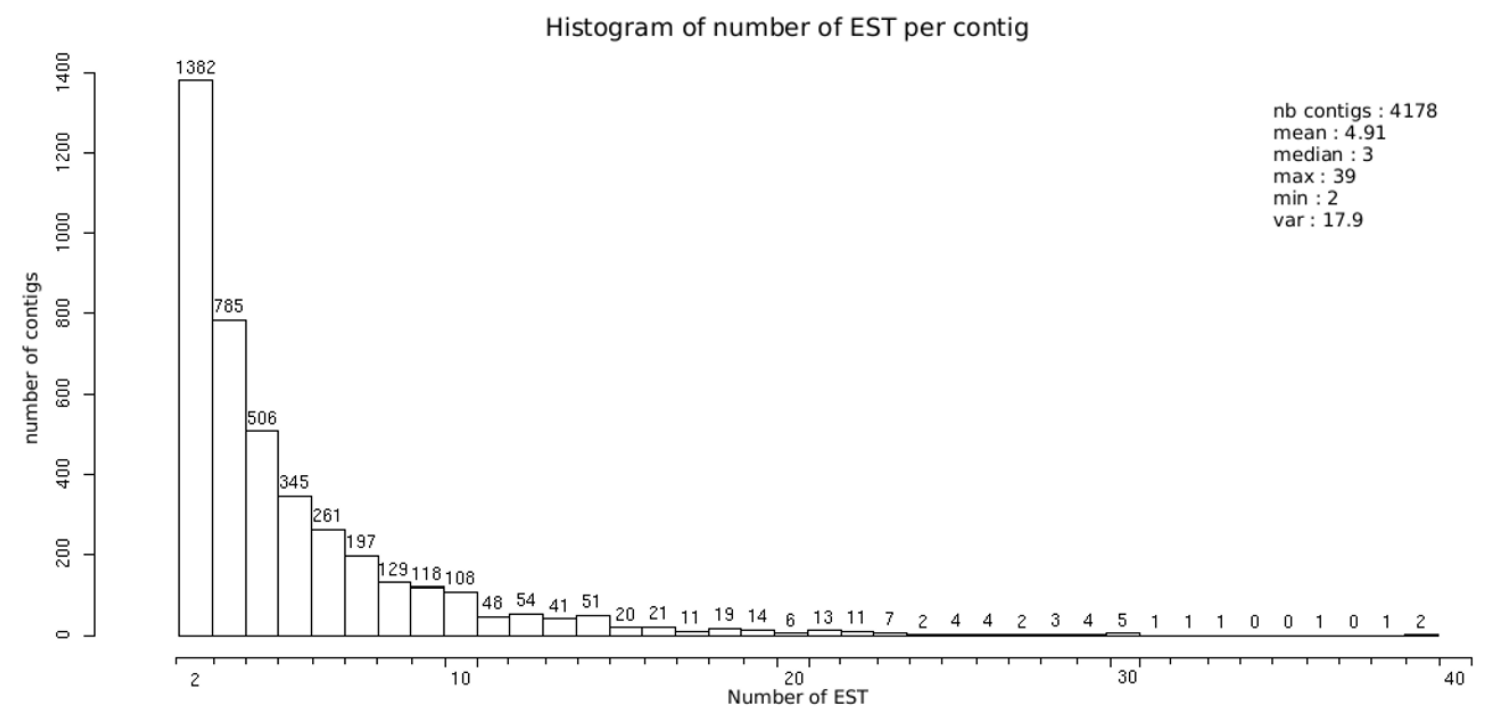

\section{Figure I}

Distribution of Microbotryum violaceum EST. EST redundancy among the 7,765 unisequences obtained from a cDNA library of the basidiomycete fungus Microbotryum vi.olaceum. The number of ESTs is indicated above each bar. 
BASE). In addition, 148 unisequences (15.31\%) showed significant similarity to transposable elements.

\section{Sequences relevant to mating-types}

Our cDNA library contained 70 sequences presenting a similarity (E-value $\leq 10^{-10}$ ) with genes belonging to, or linked to, the MAT loci in other fungi. According to the Gene Ontology classification, most of these sequences (61\%) would have molecular functions. Thirteen sequences were similar to pheromone receptors, transporters or response factors, mainly from the other basidiomycete species Coprinopsis cinerea, Schizophyllum commune, Ustilago maydis and Cryptococcus neoformans. We also identified 332 sequences similar to genes regulating mating, morphogenesis and pathogenesis, such as the mitogen-activated protein kinase (MAPK) and the cAMP dependent protein kinase (PKA), components of the PKA/ MAPK network in U. maydis [4]. Other sequences had a significant similarity to transcription factors, like the Prf1 of $U$. maydis [20], which are essential for the interconnection between the pathways of PKA and MAPK pathways.

The most interesting ESTs regarding the MAT locus of $M$. violaceum were those constituting the four unisequences similar to pheromone receptors. These four unisequences (the singlet pr0aaa87yh06 and the contigs 588, 2096 and 660) showed significant sequence similarity to each other but not enough to be assembled in a single contig. We designed primers (Table 2) within each of the four unisequences similar to pheromone receptors and performed

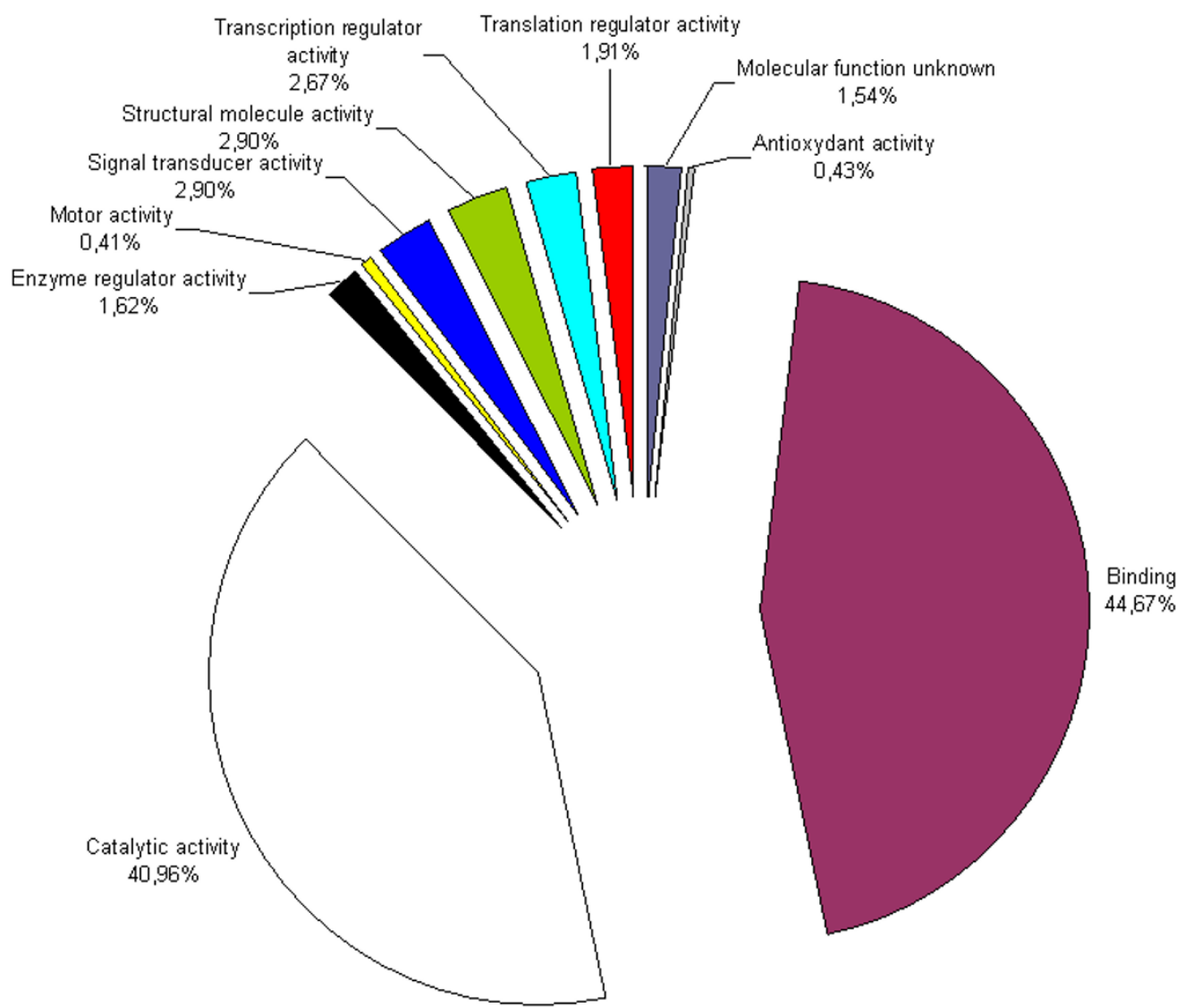

\section{Figure 2}

Molecular function categories of Microbotryum violaceum sequences. Distribution of the 797 contigs and singlets having a significant blast hit in public databases into molecular function class according to the Gene Ontology classification. 
Table I: Contigs of Microbotryum violaceum blasting significantly to the pathogenicity-related genes reported in the PHI-database

\begin{tabular}{|c|c|c|c|c|c|c|}
\hline Putative function & Contig or singlet ID & E-value & EMBL accession & PHI accession & $\begin{array}{l}\text { Gene Ontology } \\
\text { category }\end{array}$ & $\begin{array}{l}\text { Gene Ontology } \\
\text { class }\end{array}$ \\
\hline \multirow[t]{2}{*}{$A B C$-transporter } & 1816 & $6,00 \mathrm{E}-53$ & $\underline{B A C 67162}$ & 391 & $\begin{array}{c}\text { Transporter } \\
\text { activity/catalytic } \\
\text { activity }\end{array}$ & Molecular function \\
\hline & 211 & $1,00 E-16$ & AAK 15314 & 310 & $\begin{array}{c}\text { Transporter } \\
\text { activity/catalytic } \\
\text { activity }\end{array}$ & Molecular function \\
\hline Acetolactate synthase & 3362 & $8,00 \mathrm{E}-20$ & $\underline{\text { AAR29084 }}$ & 358 & Catalytic activity & Molecular function \\
\hline Adenylate cyclase & prOaaa I04yj02scm I.I & $4 \mathrm{E}-08$ & AAG60619 & 241 & Catalytic activity & Molecular function \\
\hline $\begin{array}{l}\text { ATP molecular dependent } \\
\text { chaperone }\end{array}$ & 342 & $\mathrm{I}, 00 \mathrm{E}-60$ & $\underline{\mathrm{AAA} 02743}$ & 463 & Binding & Molecular function \\
\hline $\begin{array}{l}\text { Benomyl/methotrexate } \\
\text { resistance }\end{array}$ & 547 & $2 \mathrm{E}-\mathrm{II}$ & CAA37820 & 26 & $\begin{array}{l}\text { Transporter } \\
\text { activity }\end{array}$ & Molecular function \\
\hline capsule protein & prOaaa63yn2Iscml.I & $8,00 \mathrm{E}-15$ & $\underline{\mathrm{BAC} 76819}$ & 139 & $\begin{array}{l}\text { Transporter } \\
\text { activity }\end{array}$ & Molecular function \\
\hline \multirow[t]{2}{*}{ Carnitine acetyl transferase } & 1367 & $2 \mathrm{E}-\mathrm{I} 4$ & $\underline{\mathrm{AAB} 88887}$ & 120 & Catalytic activity & Molecular function \\
\hline & 830 & IE-I2 & $\underline{\mathrm{AAB} 88887}$ & 120 & Catalytic activity & Molecular function \\
\hline \multirow[t]{3}{*}{ Chitin synthase } & 1149 & $|, 00 E-3|$ & $\underline{\mathrm{AAC} 34496}$ & 236 & Catalytic activity & Molecular function \\
\hline & pr0aaa $19 y o 09 \mathrm{scml} .1$ & $|E-| \mid$ & $\underline{A A C 35278}$ & 237 & Catalytic activity & Molecular function \\
\hline & 2395 & $9,00 \mathrm{E}-27$ & $\underline{\text { AAT77184 }}$ & 337 & Catalytic activity & Molecular function \\
\hline \multirow[t]{2}{*}{ Cyclophilin } & 229 & $9,00 \mathrm{E}-23$ & $\underline{\text { AAGI3968 }}$ & 249 & Catalytic activity & Molecular function \\
\hline & 2275 & $\mathrm{I}, 00 \mathrm{E}-20$ & AAF69795 & 213 & Catalytic activity & Molecular function \\
\hline Exopolygalacturonase PGXI & prOaaa I $2 y k 07 \mathrm{scm} \mathrm{I.I}$ & $\mathrm{I}, 00 \mathrm{E}-30$ & AAK81847 & 181 & Catalytic activity & Molecular function \\
\hline G protein alpha subunit & 3233 & $4,00 \mathrm{E}-22$ & $\underline{\text { AAC49724 }}$ & 76 & Binding & Molecular function \\
\hline Glyoxaloxidase I & 1675 & $9,00 \mathrm{E}-15$ & CAD79488 & 352 & Catalytic activity & Molecular function \\
\hline G-protein beta subunit I & 402 & $9,00 \mathrm{E}-15$ & $\underline{\text { AAP55639 }}$ & 316 & Binding & Molecular function \\
\hline $\begin{array}{l}\text { Guanine nucleotide exchange } \\
\text { factor Cdc } 24\end{array}$ & pr0aaa67yi20scm I.I & $9,00 \mathrm{E}-19$ & AAO25556 & 283 & $\begin{array}{l}\text { Enzyme regulator } \\
\text { activity }\end{array}$ & Molecular function \\
\hline \multirow[t]{2}{*}{$\begin{array}{l}\text { Guanyl nucleotide exchange } \\
\text { factor Sq12 }\end{array}$} & prOaaa26ye I Iscm I.I & $2 \mathrm{E}-\mathrm{I} 4$ & AAOI9638 & 319 & $\begin{array}{l}\text { Enzyme regulator } \\
\text { activity }\end{array}$ & Molecular function \\
\hline & prOaaa94yf22scm I.I & $6,00 \mathrm{E}-15$ & $\underline{\text { AAA02743 }}$ & 463 & Binding & Molecular function \\
\hline $\begin{array}{l}\text { Imidazole glycerol phosphate } \\
\text { dehydratase }\end{array}$ & 2572 & $3,00 \mathrm{E}-17$ & $\underline{A A B 88888}$ & 121 & $\begin{array}{l}\text { Cellular process/ } \\
\text { metabolic process }\end{array}$ & Biological process \\
\hline \multirow[t]{2}{*}{ Isocitrate lyase } & 1103 & $2,00 \mathrm{E}-39$ & AAM89498 & 261 & Catalytic activity & Molecular function \\
\hline & prOaaa36yl22scm I.I & $\mathrm{I}, 00 \mathrm{E}-36$ & AAM89498 & 261 & Catalytic activity & Molecular function \\
\hline \multirow[t]{5}{*}{ MAP Kinase } & prOaaa24yhl3scm I.I & $6,00 \mathrm{E}-29$ & $\underline{\mathrm{AAO} 27796}$ & 342 & Binding & Molecular function \\
\hline & 1219 & $4,00 \mathrm{E}-23$ & CAC47939 & 245 & Binding & Molecular function \\
\hline & 908 & $2,00 \mathrm{E}-16$ & AAK49432 & 234 & Binding & Molecular function \\
\hline & 955 & $8,00 \mathrm{E}-31$ & $\underline{\text { AAFI } 5528}$ & $|5|$ & Binding & Molecular function \\
\hline & 374 & $5,00 \mathrm{E}-25$ & $\underline{\mathrm{AAFI} 5528}$ & $|5|$ & Binding & Molecular function \\
\hline $\begin{array}{l}\text { Mitochondrial glycoprotein, } \\
\text { Mrbl }\end{array}$ & 1454 & $9,00 \mathrm{E}-19$ & AAT8II48 & 367 & $\begin{array}{l}\text { Multiorganism } \\
\text { process }\end{array}$ & Biological process \\
\hline $\begin{array}{l}\text { NADH-ubiquinone } \\
\text { oxidoreductase } 49 \mathrm{kDa} \\
\text { subunit, mitochondrial } \\
\text { precursor }\end{array}$ & 2040 & $3,00 \mathrm{E}-32$ & EAA69636 & 445 & $\begin{array}{l}\text { Multiorganism } \\
\text { process/catalytic } \\
\text { activity }\end{array}$ & $\begin{array}{l}\text { Biological process/ } \\
\text { molecular function }\end{array}$ \\
\hline $\begin{array}{l}\text { Peroxisome biogenesis - Pex } 6 \\
\text { protein }\end{array}$ & 2886 & $3,00 \mathrm{E}-31$ & $\underline{\text { AAK } 16738}$ & 226 & $\begin{array}{l}\text { Metabolic process/ } \\
\text { cellular process }\end{array}$ & Biological process \\
\hline Pheromone receptor CPRaIP & 660 & $2,00 \mathrm{E}-32$ & AAK31936 & 292 & $\begin{array}{l}\text { Signal transducer } \\
\text { activity }\end{array}$ & Molecular function \\
\hline Phosphatidylinositol 3-kinase & 4039 & $\mathrm{I}, 00 \mathrm{E}-27$ & CAA70254 & 195 & Catalytic activity & Molecular function \\
\hline
\end{tabular}


Table I: Contigs of Microbotryum violaceum blasting significantly to the pathogenicity-related genes reported in the PHI-database

\begin{tabular}{|c|c|c|c|c|c|c|}
\hline Polygalacturonase & 3187 & $2 \mathrm{E}-\mathrm{II}$ & CAA7I246 & 247 & Catalytic activity & Molecular function \\
\hline \multirow[t]{3}{*}{ Protein kinase } & 444 & $1,00 E-16$ & AAW46354 & 360 & $\begin{array}{l}\text { Binding/calalytic } \\
\text { activity }\end{array}$ & Molecular function \\
\hline & 2829 & $9,00 \mathrm{E}-48$ & $\underline{\mathrm{AAB} 68613}$ & 85 & $\begin{array}{l}\text { Binding/calalytic } \\
\text { activity }\end{array}$ & Molecular function \\
\hline & 2748 & $2,00 \mathrm{E}-23$ & AAC09291 & 158 & $\begin{array}{l}\text { Binding/calalytic } \\
\text { activity }\end{array}$ & Molecular function \\
\hline \multirow[t]{2}{*}{ Protein mannosyltransferase } & 1910 & $|, 00 E-5|$ & AAFI6867 & 452 & Catalytic activity & Molecular function \\
\hline & prOaaa54yd0Iscml.I & $8,00 \mathrm{E}-48$ & CAA67930 & 104 & Catalytic activity & Molecular function \\
\hline $\begin{array}{l}\text { Putative branched-chain } \\
\text { amino acid aminotransferase }\end{array}$ & 1888 & $9,00 \mathrm{E}-21$ & $\underline{A A D 45321}$ & 157 & Catalytic activity & Molecular function \\
\hline \multirow{4}{*}{$\begin{array}{l}\text { Rab subfamily of small } \\
\text { GTPases, Rsrlp }\end{array}$} & 231 & $8,00 \mathrm{E}-26$ & $\underline{\mathrm{CAC} 41973}$ & 339 & Binding & Molecular function \\
\hline & pr0aaa8lye23scml.I & $5,00 \mathrm{E}-22$ & $\underline{\mathrm{CAC} 41973}$ & 339 & Binding & Molecular function \\
\hline & pr0aaa $90 y b 05 \mathrm{scml} .1$ & $5 \mathrm{E}-10$ & CAC41973 & 339 & Binding & Molecular function \\
\hline & 3235 & $\mathrm{I}, 00 \mathrm{E}-44$ & CAC41973 & 339 & Binding & Molecular function \\
\hline $\begin{array}{l}\text { Ras-like small GTPases } \\
\text { CaRhol }\end{array}$ & 3583 & $4,00 \mathrm{E}-38$ & BAA24262 & 270 & Binding & Molecular function \\
\hline Topoisomerase I & 1694 & $\mathrm{I}, 00 \mathrm{E}-\mathrm{I} 7$ & $\underline{A A B 39507}$ & 80 & $\begin{array}{l}\text { Binding/catalytic } \\
\text { activity }\end{array}$ & Molecular function \\
\hline \multirow[t]{2}{*}{ Transcriptional repressor } & prOaaal lyo22scml.I & $2,00 \mathrm{E}-20$ & $\underline{\mathrm{AAB} 63195}$ & 211 & $\begin{array}{l}\text { Cellular process/ } \\
\text { metabolic process }\end{array}$ & Biological process \\
\hline & prOaaa $104 y m 01$ scm I.I & $9,00 \mathrm{E}-20$ & $\underline{\mathrm{AAB} 63195}$ & 211 & $\begin{array}{l}\text { Cellular process/ } \\
\text { metabolic process }\end{array}$ & Biological process \\
\hline \multirow[t]{8}{*}{ Transmembrane protein } & 631 & $5,00 \mathrm{E}-42$ & $\underline{A A D 51594}$ & 267 & Binding & Molecular function \\
\hline & pr0aaa92ybl9scml.I & $5,00 \mathrm{E}-32$ & $\underline{\text { AAD51594 }}$ & 267 & Binding & Molecular function \\
\hline & 2916 & $7,00 \mathrm{E}-25$ & $\underline{\text { AAD51594 }}$ & 267 & Binding & Molecular function \\
\hline & pr0aaa47ydI IscmI.I & $7,00 \mathrm{E}-22$ & AAD51594 & 267 & Binding & Molecular function \\
\hline & 1972 & $3,00 \mathrm{E}-19$ & $\underline{\text { AAD51594 }}$ & 267 & Binding & Molecular function \\
\hline & 3153 & $\mid E-13$ & $\underline{\text { AAD51594 }}$ & 267 & Binding & Molecular function \\
\hline & pr0aaa62yh02scml.I & $7 E-13$ & $\underline{\text { AAD51594 }}$ & 267 & Binding & Molecular function \\
\hline & 2016 & $2 \mathrm{E}-\mathrm{II}$ & AAD5I594 & 267 & Binding & Molecular function \\
\hline \multirow{2}{*}{$\begin{array}{l}\text { Trehalose-6-phosphate } \\
\text { phosphatase }\end{array}$} & 2473 & $9,00 \mathrm{E}-49$ & AAN46744 & 322 & Catalytic activity & Molecular function \\
\hline & 960 & $2,00 \mathrm{E}-21$ & AAN46744 & 322 & Catalytic activity & Molecular function \\
\hline Uac & pr0aaa75ycl4scml.I & $9 \mathrm{E}-\mathrm{I} 4$ & AAA57469 & 22 & Catalytic activity & Molecular function \\
\hline \multirow[t]{2}{*}{ Urease } & pr0aaa84yg09scm I.I & $3 \mathrm{E}-13$ & $\underline{A A C 62257}$ & 194 & Metabolic process & Biological process \\
\hline & 1663 & $4 \mathrm{E}-\mathrm{II}$ & AAC62257 & 194 & Metabolic process & Biological process \\
\hline vacuolar $\left(\mathrm{H}^{+}\right)$-ATPase subunit & 2474 & $2 \mathrm{E}-\mathrm{I} 3$ & AAK8I705 & 235 & Localization & $\begin{array}{l}\text { Cellular } \\
\text { component }\end{array}$ \\
\hline \multirow{3}{*}{$\begin{array}{l}\text { Virulence associated DEAD } \\
\text { box protein I }\end{array}$} & 1516 & $3 \mathrm{E}-13$ & $\underline{A A V 41010}$ & 423 & Binding & Molecular function \\
\hline & 2939 & $4 \mathrm{E}-12$ & AAV41010 & 423 & Binding & Molecular function \\
\hline & pr0aaa70ym I8scml.I & $8 \mathrm{E}-\mathrm{II}$ & $\underline{A A V 41010}$ & 423 & Binding & Molecular function \\
\hline \multirow[t]{3}{*}{ Hypotethical protein } & 1112 & $\mathrm{I}, 00 \mathrm{E}-28$ & EAL03139 & 290 & Binding & Molecular function \\
\hline & 1224 & $4,00 \mathrm{E}-16$ & EAL03139 & 290 & Binding & Molecular function \\
\hline & prOaaal lyc08scm I.I & $7 E-13$ & EAL03139 & 290 & Binding & Molecular function \\
\hline
\end{tabular}


PCRs on A1 and A2 sporidial lines of ten strains of M. violaceum. Amplification products were of higher size than expected from ESTs for 3 of the unisequences, indicating the presence of introns (Table 2). The amplifications corresponding to each of the four unisequences were specific of a single mating type (Table 2).

Furthermore, the p-distance [21] showed that sequences of singlet pr0aaa87yh06 and contig 588 were highly similar ( $p=0.273)$ and identical on the second halves of the sequences $(p=0.000)$. Inspection of the chromatograms showed that one of the 3 ESTs assembled in the contig 588 was of very poor quality on the first half of the sequences, suggesting that the singlet pr0aaa87yh06 and contig 588 were actually probably transcripts of the same gene. This was checked by designing primers on the most different parts of the two unisequences, which amplification products indeed yielded identical sequences, including the intronic parts.

These two sequences were less similar to the contigs 2096 and 660 ( $\mathrm{p}=0.702$ and 0.793 respectively). The contigs 2096 and 660 overlapped only on 25 bp, but aligned one to each other perfectly at their edges $(p=0.000)$, suggesting that they represent ESTs from the same gene. Contigs
2096 and 660 were not assembled into a single contig because the region of overlap with sufficient PHRED quality sequence was too short. The PCR performed using the forward primer of the Contig 2096 and the reverse primer of the Contig 660 (Table 2) yielded a single amplification product whose sequence read without apparent heterogeneity on the chromatograms. This indicates that the contigs 2096 and 660 indeed correspond to the same pheromone receptor.

Microbotryum violaceum thus appears to carry a single pheromone receptor at the $\mathrm{A} 1$ locus and a single pheromone receptor at the $\mathrm{A} 2$ locus, which would be in agreement with its bipolar status. In contrast, tetrapolar species such as $C$. cinereus and $S$. commune have several pheromone receptors at each of the alternate forms of the $\mathrm{B}$ mating type locus [22,23]. A genome walking approach allowed us to obtain the complete sequence of the putative A1 and A2 pheromone receptors of Microbotryum violaceum (Figure 3A; Genbank accession numbers EF584742 and EF584741, respectively for the $\mathrm{A} 1$ and $\mathrm{A} 2$ pheromone receptors).

The putative pheromone receptors identified in our cDNA library did not show highly significant similarity to the

Table 2: Unisequences of Microbotryum violaceum blasting against pheromone receptors. For each of the four unisequences significantly blasting against pheromone receptors: best hits, primer designed for PCR amplification, expected size from the EST sequence, rough amplification size obtained, and mating type of the sporidia that gave amplification products.

\begin{tabular}{|c|c|c|c|c|c|c|}
\hline & $\begin{array}{c}\text { Number of } \\
\text { ESTs }\end{array}$ & Best hits & Primers & $\begin{array}{l}\text { Contig size } \\
\text { obtained from } \\
\text { ESTs }\end{array}$ & $\begin{array}{c}\text { Rough } \\
\text { amplification } \\
\text { size }\end{array}$ & $\begin{array}{c}\text { Amplification in } \\
\text { sporidia of } \\
\text { mating type }\end{array}$ \\
\hline \multirow[t]{2}{*}{ Contig588 } & 3 & $\begin{array}{l}\text { Rcbl of Coprinopsis } \\
\text { cinerea }\end{array}$ & $\begin{array}{l}\mathrm{F}^{\prime}: \\
\text { GGAAGGCCATTACAA } \\
\text { GAAAGG }\end{array}$ & 350 & 500 & A2 only \\
\hline & & $\begin{array}{l}\text { Bbr2 of } \\
\text { Schizophyllum } \\
\text { commune }\end{array}$ & $\begin{array}{l}\mathrm{R}^{2}: \\
\text { TGTGCTTTTCGCTCT } \\
\text { TAGCA }\end{array}$ & & & \\
\hline \multirow[t]{2}{*}{ Contig660 } & 4 & Rcb2 of $C$. cinerea & $\begin{array}{l}\text { F: } \\
\text { ACGATTCCAGTAGGC } \\
\text { GTGAA }\end{array}$ & 551 & 800 & Al only \\
\hline & & $\begin{array}{l}\text { B alpha } 8 \text { of } S . \\
\text { commune }\end{array}$ & $\begin{array}{l}\text { R: } \\
\text { CTGCGTCACGATACC } \\
\text { TTTCTT }\end{array}$ & & & \\
\hline \multirow[t]{2}{*}{ Contig2096 } & 3 & $\begin{array}{l}\text { Bbr2 of } S . \\
\text { commune }\end{array}$ & $\begin{array}{l}\text { F: } \\
\text { TCCTTTGTCACGACA } \\
\text { AGCAC }\end{array}$ & 213 & 220 & Al only \\
\hline & & Rcb3 of $C$. cinerea & $\begin{array}{l}\text { R: } \\
\text { CCAATTTTCACGCCT } \\
\text { ACTGG }\end{array}$ & & & \\
\hline \multirow[t]{2}{*}{$\begin{array}{l}\text { Singlet } \\
\text { pr0aaa87yh06 }\end{array}$} & I & Rcbl of $C$. cinerea & $\begin{array}{l}\text { F: } \\
\text { ATCAGAATATGACGG } \\
\text { CAGCA }\end{array}$ & 383 & 600 & A2 only \\
\hline & & $\begin{array}{l}\text { Bbr2 of } S . \\
\text { commune }\end{array}$ & $\begin{array}{l}\text { R: } \\
\text { AAGAAAGGGAACTCC } \\
\text { AAATGC }\end{array}$ & & & \\
\hline
\end{tabular}

I F: Forward primer

2 R: Reverse primer 
pheromone receptors of $U$. maydis and $U$. hordei, which explains why they hybridized only weakly on Southern blots [24], and why cloning attempts of the M. violaceum mating type locus by designing degenerate primers from the $U$. maydis sequences have failed (T. Giraud, unpublished).

The cloning of the complete mating type locus of M. violaceum is currently under way, starting from the pheromone receptors obtained in the present library. The complete sequence of the mating-type locus will allow identifying the organisation and composition of this genomic region, and thus understand how the transition occurred between tetrapolarity and bipolarity in M. violaceum or its ancestral lineages. One tentative hypothesis given the data at hand is that it exists a single allele of each mating type locus and that the two mating type loci are linked, as in U. hordei [7]. Recombination is indeed sup- pressed along most of the sex chromosomes in M. violaceum [25].

\section{Other sequences relevant to pathogenesis}

A total of 70 sequences had a high similarity to genes shown experimentally to play a role in pathogenicity in other fungi (Table 2). An important class of proteins in pathogenicity is the secretome, which play important roles in penetration and colonization of plant tissues [26]. No sequence in MICROBASE presented high similarity with genes encoding cell wall-degrading enzymes, such as lyases, lipases, proteases, and we detected only two polygalacturonases. Plant pathogens that kill host cells, like Magnaporthe grisea and Fusarium graminearum, contain in their genome many genes involved in degradation of cell tissue. In contrast, it is not surprising to find a reduced number of genes involved in such hydrolytic functions in fungi with a biotrophic life style in which host damage is

A)

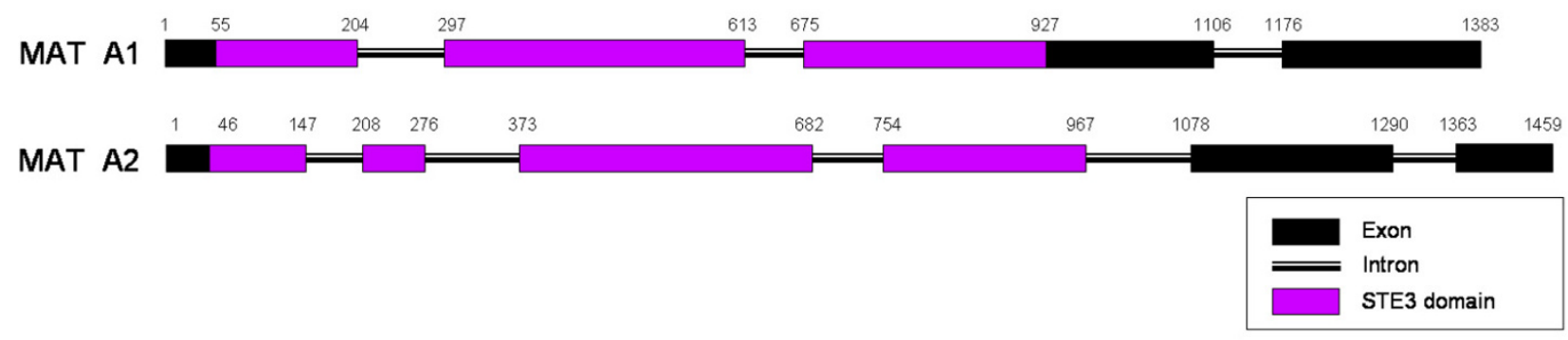

B)

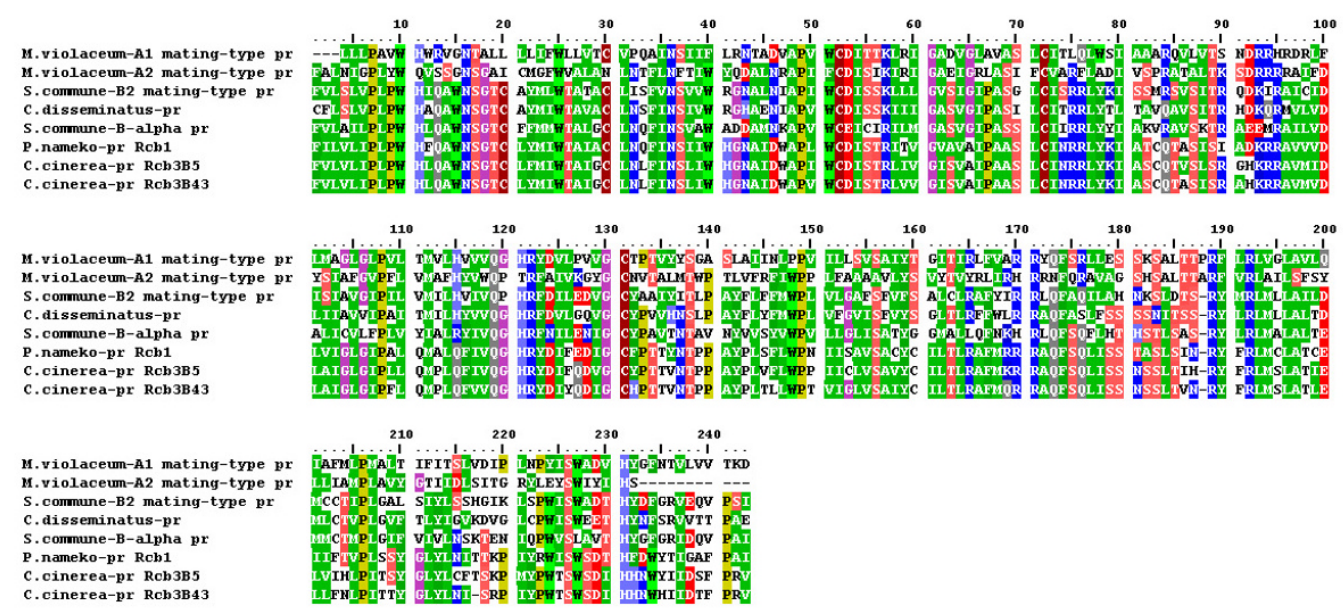

\section{Figure 3}

Putative pheromone receptors in Microbotryum. violaceum. A) Diagram of the two putative pheromone receptor genes identified in the EST library of Microbotryum violaceum, respectively linked to the AI and A2 mating type. B) Alignment of the two putative pheromone receptors of Microbotryum violaceum with the most similar published protein sequences of other fungi: B2 and B-alpha of Schizophyllum commune, the transmembrane pheromone receptor of Coprinellus disseminatus and Rcb3B5 of Coprinopsis cinerea. 
minimized, like M. violaceum. Similar conclusions have been drawn from the complete genome sequence of Ustilago maydis [27], which also has a biotrophic life style. The genome of $U$. maydis contained in contrast numerous secreted proteins with unknown functions, and even with no similarity to any other proteins in the databases. The total number (594) of proteins predicted to be secreted in MICROBASE was similar to that in the genome of $U$. maydis [27], and the percentage of secreted proteins without a significant hit in databases was also very high $(86.4 \%$ in MICROBASE). This suggests that the specific and intimate relationships between biotrophic fungi and their host plant select for specific secreted functions.

In several fungi, the CAMP signalling and two MAP kinase pathways have been implicated in regulating various plant infection processes, in particular in monocot-infecting smuts [28]. Several contigs of $M$. violaceum were similar to enzymes of these molecular pathways, including $G$ proteins, protein kinases and Ras proteins. In U. maydis for instance, disruption of Ras 2 resulted in loss of pathogenicity and dramatic changes in cell morphology [29]. Another important molecular pathway in pathogenic fungi is the Calcineurin/cyclophilin signalling [30], for which we also detected putative genes in the MICROBASE. Other important molecules involved in pathogeniticy belong to the secondary metabolism which includes P450 genes, such as the putative ones present in the MICROBASE, or the small peptides synthetized by nonribosomal peptide synthases (NRPS). We detected contigs similar to NRPS, such as the one similar to CPS1 [31].

\section{Expressed transposable elements}

Our library presented 148 unisequences with significant similarity to transposable elements (TE), with an additional 10 showing putative or weak similarity to TEs. The 148 unisequences, when categorized by the major types of Class I (RNA-based replication) and Class II (DNA-based replication) transposable elements, were in similar relative frequencies as TEs from the M. violaceum genomic survey [12] (Figure 4). Putative Class II DNA transposons and a maturase sequence from a mitochondrial Group II Intron were also identified among the expressed sequences, but were not found in the prior genomic survey. Although Hood et al. [32] showed that the RIP (repeat-induced point mutation) genome defense has been very active in $M$. violaceum, our results suggest that the transposable elements can escape this genomic mechanism of defense to some extent, at least regarding the transcriptional activity. In fact, there is evidence of RIP mutation among the expressed TE sequences; five unisequences could be aligned with the genomic consensus of copia-like integrase gene from a prior analysis of RIP in $M$. violaceum [32], and among these alignments mutations at

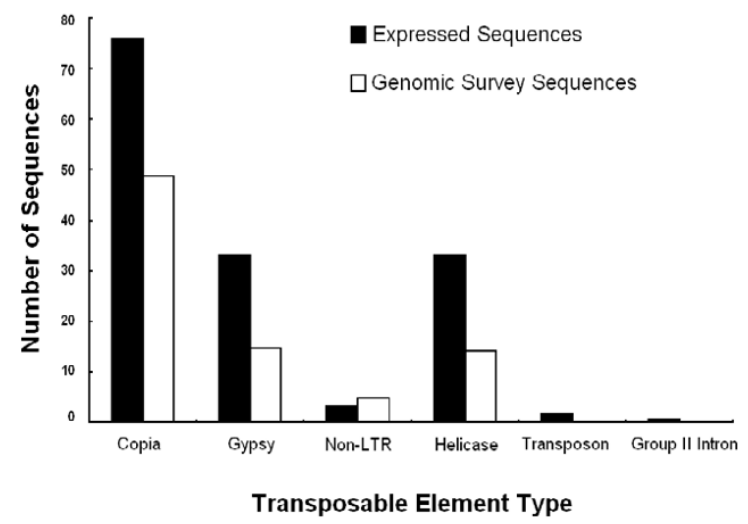

Figure 4

Comparison of expressed and genomic copies of Microbotryum violaceum transposable elements. Class I elements are represented by the Copia, Gypsy, and NonLTR (long-terminal repeat) categories; Class II elements are represented by the Helicase and DNA Transposon categories. The Group II Intron category corresponds to a mitochondrial mobile element. The data on genomic survey sequences are from ref [12].

RIP recognition sites were 2 to 3 times more frequent that to any other sites.

Prior studies have reported that some unidentified transposable elements may be active only during mitosis, whereas others would be active during meiosis [33], and the conditions under which our library was built may therefore lead to an underestimation of the TE transcriptional activity. A more specific study is required to understand the importance of the RIP mechanism in the accumulation of transposable elements in the genome of M. violaceum, especially as RIP-affected and non-functional TE copies may still be transcribed. The comparison of TE transcripts in the MICROBASE with the genomic copies should be interesting to estimate the impact of the RIP defense mechanism in M. violaceum. We did not identify any EST similar to the RID (RIP defective) DNA methyltransferase gene required for RIP in Neurospora crassa [34], although we detected several sequences similar to methyltransferases.

\section{Conclusion}

This study, providing the first extensive genomic dataset on $M$. violaceum, has permitted the detection of many genes putatively involved in mating, some of which were shown to be linked to the mating-type locus, and also many genes possibly involved in pathogenesis. Studies of reverse genetics are however required to validate these 
putative biological functions. Studies of comparative genomics among fungi should also benefit from the existence of resources such as the MICROBASE [35]. This extensive database will not only allow comparing the sequence evolution among species, but also searches for the presence of genes and the numbers for gene families. Such comparative approaches yield valuable insights into the evolution of host-pathogen interactions [35]. Furthermore, it is now possible to clone and sequence the whole mating type locus of $M$. violaceum, allowing elucidating its organization. Comparison with the mating type loci of other basidiomycetes will then provide insights into its evolution, in particular into the mechanism of the transition between tetra- and bipolarity. Finally, the high expression level of transposable elements raises questions about the importance of the RIP genome defense, and how it can be escaped.

\section{Methods}

Microbotryum violaceum strain and culture conditions

Teliospores from the strain 100.02 of M. violaceum, collected from the host Silene latifolia in 2001 in the Alps, near Tirano in Italy, was plated on GMB1 medium [36]. On such nutritive media, diploid teliospores germinate and produce haploid sporidia of the two mating type A1 and A2. A1 and A2 sporidia lines from the strain 100.02 were identified by pairing with existing stocks of known mating type.

A mixed suspension of A1 and A2 sporidia ( $250 \mu \mathrm{L}$ of each) was plated on water agar supplemented with $\alpha$ tocopherol $(10 \mathrm{IU} / \mathrm{g})$ and incubated at $4{ }^{\circ} \mathrm{C}$ for one week. These conditions of low nutrients with $\alpha$-tocopherol are thought to mimic the host plant surface for the fungus, because sporidia conjugate and produce hyphae of a few cells [37]. This was checked using a light microscope (400×).

RNA isolation, CDNA library construction and sequencing Total RNA was extracted from conjugated cells and hyphae using the Trizol reagent following the manufacture protocol (Invitrogen, The Netherlands). Extractions yielded $50 \mu \mathrm{g}-500 \mu \mathrm{g}$ of total RNA. Poly $\left(\mathrm{A}^{+}\right) \mathrm{RNA}$ was purified using the mRNA Absolutely Purification Kit (Stratagene, CA). The SuperSmart cDNA Synthesis Kit (Clontech, CA) was used to synthesize CDNA, and the library was normalized using the Trimmer kit (Evrogen, Moscow) that reduces the quantity of the most abundant cDNA copies. cDNAs were ligated into the pGEM-T vector (Promega, WI). To test the quality of the ligation, we transformed ultracompetent cells (XL10-Gold, Stratagene, CA) and amplified inserts from 100 clones. The average size of inserts was $500 \mathrm{bp}$. Individual colonies were examined using the blue-white selection for the vector giving $>50 \%$ of vector with inserts and an estimate of $2.0 \times 10^{5}$ cfu. Forty thousand clones were then sequenced in one direction by the Genoscope (Evry-France) using the primer of cDNA synthesis kit (SMART II A Oligonucleotide 5'-AAG CAG TGG TAT CAA CGC AGA GTA CGC GGG-3').

\section{Sequence analyses and EST clustering}

Raw sequence data were cleaned from vector and adaptor sequences. Contaminating plasmid sequences, such as $E$. coli, mitochondrial or ribosomal fungal sequences were removed from the analyses. PHRED software $[38,39]$ was used for base-calling the chromatogram trace files. Only sequences with a PHRED score over 20 on at least $100 \mathrm{bp}$ were released in the EST division of the EMBL-EBI (European Molecular Biology Laboratory - European Bioinformatic Institute) Nucleotide Sequence Database.

ESTs were aligned and assembled into contigs using the CAP3 software [40] when the criterion of a minimum identity of $95 \%$ over 50 bp was met. When an EST could not be assembled with others in a contig, it remained as a "singlet". The contigs and the singlets should thus correspond to sequences of unique genes, and will be called hereafter "unisequences". The consensus sequences of the contigs and the sequences of the singlets were compared to the sequences in the GenBank database and in the Uniprot database using the tBLASTx and the BLASTx algorithms [41]. Unisequences showing significant similarity (E-value $<=10^{-4}$ ) to database entries were annotated using the most significant matches. Unisequences were also classified into Gene Ontology functional categories [42] based on BLAST similarities to known genes of the NCBI $\mathrm{nr}$ (non-redundant) protein database and using the Blast2GO annotation tool [43]. Sequences were also compared to the pathogenicity genes assembled in the PHI database $[2,44]$ and to the genes linked to the mating-type in other fungi using a manually built list of such genes. The sequences showing significant similarity to transposable elements were also recorded. WoLF PSORT version 2.0 [45] was used to predict protein localization using the higher prediction score for external compartments. Finally, using a modified version of the ESTIMA tool [46] we developed a public database named MICROBASE, dedicated to Microbotryum violaceum EST management and analysis. This database includes information on EST sequences, contigs, annotations, gene ontology functional categories and search programs to compare similarities of any sequence against the database. MICROBASE is accessible freely through a web interface [18].

\section{Amplification of putative pheromone receptors}

Primers were designed in the four unisequences with significant sequence similarity to pheromone receptors (Table 2) and amplifications were performed on DNA extracted from sporidia of known mating type, from ten 
different strains of $M$. violaceum, of various geographical origins. DNA was extracted from single-sporidial colonies using the Chelex (Biorad) protocol [47]. PCR amplifications were performed using a PTC 100 thermal cycler (MJ Research), with $65^{\circ} \mathrm{C}$ as the annealing temperature, for the amplification to be as specific as possible, using the Qbiogene (Irvine, CA) Taq polymerase following the manufacturer recommendations.

\section{Genome walking}

High quality genomic DNA was isolated from a Microbotryum violaceum strain from $S$. latifolia. The DNA was digested by blunt end cutting enzymes (DraI, PvuII, EcoRV and StuI) provided in the Universal GenomeWalker kit (BD Biosciences, Clontech, USA). The digested DNA was then purified and ligated overnight with the adaptors provided in the kit. The genome walking approach was followed according to the manufacturer instructions.

\section{Authors' contributions}

TG and RY contributed to the conception and design of the study, to the acquisition and analysis of data, to coordination of the study, and were involved in drafting the manuscript. SM, HC, MEH participated in data analysis and drafting of the manuscript. FR and AG were involved in data analysis. CR and PW carried out the sequencing and first data analysis. BD performed sequences and analyses of the pheromone receptors. All authors read and approved the final manuscript.

\section{Acknowledgements}

We thank Jessie Abbate for the genome walking libraries and Bernard Lejeune for helpful discussions and advice. Muriel Viaud, Bernard Lejeune and Marc-Henri Lebrun provided helpful comments on an earlier draft of the manuscript. Joelle Amselem provided help in sequence analysis. This work was funded by $\mathrm{ACl}$ Jeunes Chercheurs and by the "Consortium National de Recherche en Génomique" for sequencing the library.

\section{References}

I. Xu J: Microbial ecology in the age of genomics and metagenomics: concepts, tools, and recent advances. Molecular Ecology 2006, I5:1713-1729.

2. Winnenburg R, Baldwin TK, Urban M, Rawlings C, Kohler J, Hammond-Kosack KE: PHI-base: a new database for pathogen host interactions. Nucl Acids Res 2006, 34:D459-464.

3. Brachmann A, Weinzierl G, Kamper J, Kahmann R: Identification of genes in the $b W / b E$ regulatory cascade in Ustilago maydis. Molecular Microbiology 200I, 42: I047-I063.

4. Feldbrugge M, Kamper J, Steinberg G, Kahmann R: Regulation of mating and pathogenic development in Ustilago maydis. Current Opinion in Microbiology 2004, 7:666-672.

5. Raper JR: Genetics of sexuality in higher fungi. New York, Ronald Press; 1966.

6. Raper JR, Flexer AS: Mating systems and evolution of the Basidiomycetes. In Evolution in the Higher Basidiomycetes Edited by: Petersen RH. Knoxville, TN., University of Tennessee Press; 1971:149-167.

7. Bakkeren G, Kronstad JW: Linkage of mating-type loci distinguishes bipolar from tetrapolar mating in Basidiomycetous smut fungi. Proc Natl Acad Sci U S A 1994, 9 I (15):7085-7089.

8. James TY, Srivilai P, Kues U, Vilgalys R: Evolution of the bipolar mating system of the mushroom Coprinellus disseminatus from its tetrapolar ancestors involves loss of mating-type- specific pheromone receptor function. Genetics 2006, I72:1877-|89|.

9. Hibbett DS, Donoghue MJ: Analysis of character correlations among wood decay mechanisms, mating systems, and substrate ranges in homobasidiomycetes. Systematic Biology 200I, 50:215-242.

10. Thrall PH, Biere A, Antonovics J: Plant-life history and disease susceptibility - the occurrence of Ustilago violacea on different species within the Caryophyllaceae. Journal of Ecology 1993 , $81: 489-498$

II. Day AW: Mating type and morphogenesis in Ustilago violacea. Bot Gaz 1979, 140:94-109.

12. Hood ME: Repetitive DNA in the automictic fungus Microbotryum violaceum. Genetica 2005, 124: I-10.

13. Wang L, Perlin MH: Isolation of a novel gene (HSGcl I) whose expression is apparently limited to the hyphal stage of Microbotryum violaceum. International Journal of Plant Sciences 1998, I59:206-212

14. Sacadura NT, Saville BJ: Gene expression and EST analyses of Ustilago maydis germinating teliospores. Fungal Genet Biol 2003, 40(I):47-64.

15. Viaud M, Legeai F, Pradier JM, Brygoo Y, Bitton F, Weissenbach J, Brunet-Simon A, Duclert A, Fillinger S, Fortini D, Gioti A, Giraud C, Halary S, Lebrun I, Le Pecheur P, Samson D, Levis C: Expressed sequence tags from the phytopathogenic fungus Botrytis cinerea. European Journal of Plant Pathology 2005, I I I: I39- I 46.

16. Panabieres F, Amselem J, Galiana E, Le Berre JY: Gene identification in the oomycete pathogen Phytophthora parasitica during in vitro vegetative growth through expressed sequence tags. Fungal Genet Biol 2005, 42:6 I I-623.

17. Galagan JE, Henn MR, Ma LJ, Cuomo CA, Birren B: Genomics of the fungal kingdom: Insights into eukaryotic biology. Genome Research 2005, 15:|620-1631.

18. Database MICROBASE [http://genome.jouy.inra.fr/microbase]

19. Skinner W, Keon J, Hargreaves J: Gene information for fungal plant pathogens from expressed sequences. Current Opinion in Microbiology 200I, 4:38I-386.

20. Hartmann HA, Kahmann R, Bolker M: The pheromone response factor coordinates filamentous growth and pathogenicity in Ustilago maydis. Embo Journal 1996, I5:1632-1641.

21. Nei M, Kumar S: Molecular evolution and phylogenetics. New York, Oxford University Press; 2000.

22. Fowler TJ, Mitton MF, Vaillancourt LJ, Raper CA: Changes in mate recognition through alterations of pheromones and receptors in the multisexual mushroom fungus Schizophyllum commune. Genetics 200I, I58:149I-I503.

23. Halsall JR, Milner MJ, Casselton LA: Three subfamilies of pheromone and receptor genes generate multiple $B$ mating specificities in the mushroom Coprinus cinereus. Genetics 2000, 154:1II5-II23.

24. Bakkeren G, Gibbard B, Yee A, Froeliger E, Leong S, Kronstad J: The a-loci and b-loci of Ustilago maydis hybridize with DNAsequences from other smut fungi. Molecular Plant-Microbe Interactions 1992, 5:347-355.

25. Hood ME, Antonovics J, Koskella B: Shared forces of sex chromosome evolution in haploid-mating and diploid-mating organisms: Microbotryum violaceum and other model organisms. Genetics 2004, 168:141-146.

26. Kars I, van Kan JAL: Intracellular enzymes and metabolites involved in pathogenesis of Botrytis. In Botrytis: Biology, Pathology and Control Edited by: Elad Y, Williamson B, Tudzynski P and Delen N. , Kluwer Academic Publisher; 2004.

27. Kamper J, Kahmann R, Bolker M, Ma LJ, Brefort T, Saville BJ, Banuett F, Kronstad JW, Gold SE, Muller O, Perlin MH, Wosten HAB, de Vries R, Ruiz-Herrera J, Reynaga-Pena CG, Snetselaar K, McCann M, Perez-Martin J, Feldbrugge M, Basse CW, Steinberg G, Ibeas JI, Holloman W, Guzman P, Farman M, Stajich JE, Sentandreu R, Gonzalez-Prieto JM, Kennell JC, Molina L, Schirawski J, Mendoza-Mendoza A, Greilinger D, Munch K, Rossel N, Scherer M, Vranes M, Ladendorf O, Vincon V, Fuchs U, Sandrock B, Meng S, Ho ECH, Cahill MJ, Boyce KJ, Klose J, Klosterman SJ, Deelstra HJ, Ortiz-Castellanos L, Li WX, Sanchez-Alonso P, Schreier PH, Hauser-Hahn I, Vaupel M, Koopmann E, Friedrich G, Voss H, Schluter T, Margolis J, Platt D, Swimmer C, Gnirke A, Chen F, Vysotskaia V, Mannhaupt G, Guldener U, Munsterkotter M, Haase D, Oesterheld M, Mewes HW, Mauceli EW, DeCaprio D, Wade CM, Butler J, Young S, Jaffe DB, Calvo S, Nus- 
baum C, Galagan J, Birren BW: Insights from the genome of the biotrophic fungal plant pathogen Ustilago maydis. Nature 2006, 444:97-10I.

28. Lee N, D'Souza CA, Kronstad JW: Of smuts, blasts, mildews, and blights: cAMP signaling in phytopathogenic fungi. Annu Rev Phytopathol 2003, 41:399-427.

29. Lee N, Kronstad JW: ras2 Controls morphogenesis, pheromone response, and pathogenicity in the fungal pathogen Ustilago maydis. Eukaryotic Cell 2002, I:954-966.

30. Viaud M, Brunet-Simon A, Brygoo Y, Pradier JM, Levis C: Cyclophilin $A$ and calcineurin functions investigated by gene inactivation, cyclosporin $A$ inhibition and cDNA arrays approaches in the phytopathogenic fungus Botrytis cinerea. Molecular Microbiology 2003, 50: I 45 I- I 465.

3I. Lu SW, Kroken S, Lee BN, Robbertse B, Churchill ACL, Yoder OC, Turgeon BG: A novel class of gene controlling virulence in plant pathogenic ascomycete fungi. Proc Natl Acad Sci U S A 2003, 100:5980-5985.

32. Hood ME, Katawczik M, Giraud T: Repeat-induced point mutation and the population structure of transposable elements in Microbotryum violaceum. Genetics 2005, 170:108I-1089.

33. Garber ED, Ruddat M: Genetics of Ustilago violacea. XXXIV. Genetic evidence for a transposable element functioning during mitosis and two transposable elements functioning during meiosis. International Journal of Plant Sciences 1998, 159:1018-1022.

34. Freitag M, Williams RL, Kothe GO, Selker EU: A cytosine methyltransferase homologue is essential for repeat-induced point mutation in Neurospora crassa. Proc Natl Acad Sci U S A 2002, 99:8802-8807.

35. Xu JR, Peng YL, Dickman MB, Sharon A: The dawn of fungal pathogen genomics. Annu Rev Phytopathol 2006, 44:337-366.

36. Thomas A, Shykoff J, Jonot O, Giraud T: Sex-ratio bias in populations of the phytopathogenic fungus Microbotryum violaceum from several host species. International Journal of Plant Sciences 2003, 164:641-647.

37. Day AW, Garber ED: Ustilago violacea, anther smut of the Caryophyllaceae. Advances in Plant Pathology 1988, 6:457-482.

38. Ewing $B$, Green P: Base-calling of automated sequencer traces using phred. II. Error probabilities. Genome Research 1998, 8:186-194.

39. Ewing B, Hillier L, WendI MC, P. G: Base-calling of automated sequencer traces using phred. I. Accuracy assessment. Genome Research 1998, 8:175-185.

40. Huang X, Madan A: CAP3: A DNA Sequence Assembly Program. Genome Res 1999, 9:868-877.

41. Altschul SF, Madden TL, Schäffer AA, Zhang J, Zhang Z, Miller W, Lipman D]: Gapped BLAST and PSI-BLAST: a new generation of protein database search programs. Nucleic Acids Research 1997, 25:3389-3402.

42. Database Gene Ontology [http://www.geneontology.org]

43. Conesa A, Gotz S, Garcia-Gomez JM, Terol J, Talon M, Robles M: Blast2GO: a universal tool for annotation, visualization and analysis in functional genomics research. Bioinformatics 2005, 21:3674-3676

44. PHI database [http://www.phi-base.org]

45. Horton P, Park KJ, Obayashi T, Nakai K: WoLF PSORT: protein localization predictor. Nucleic Acids Res 2007, 35(Web Server issue):W585-7.

46. Kumar CG, LeDuc R, Gong G, Roinishivili L, Lewin HA, Liu L: ESTIMA, a tool for EST management in a multi-project environment. BMC Bioinformatics 2004, 5: 176-187.

47. Bucheli E, Gautschi B, Shykoff JA: Differences in population structure of the anther smut fungus Microbotryum violaceum on two closely related host species, Silene latifolia and S. dioica. Molecular Ecology 200 I, I 0:285-294.

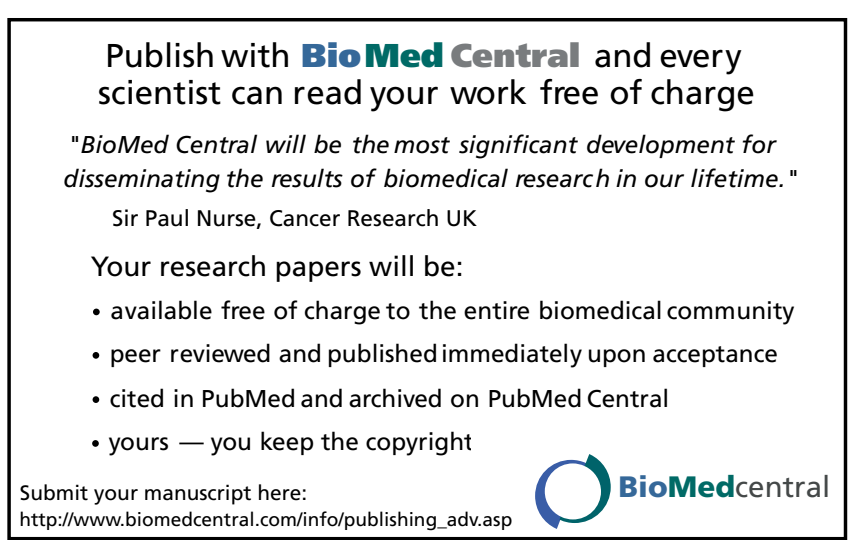

